\title{
Arnault SKORNICKI et Jérôme TOURNADRE, La Nouvelle Histoire des idées politiques
}

\section{Giovanni Carpinelli}

\section{(2) OpenEdition}

1 Journals

\section{Édition électronique}

URL : http://journals.openedition.org/ress/3156

DOI : 10.4000/ress.3156

ISBN : $1663-4446$

ISSN : $1663-4446$

Éditeur

Librairie Droz

Édition imprimée

Date de publication : 15 novembre 2015

Pagination : 273-276

ISSN : 0048-8046

Référence électronique

Giovanni Carpinelli, « Arnault SKORNICKI et Jérôme TOURNADRE, La Nouvelle Histoire des idées politiques », Revue européenne des sciences sociales [En ligne], 53-2 | 2015, mis en ligne le 15 novembre 2015, consulté le 22 septembre 2020. URL : http://journals.openedition.org/ress/3156 ; DOI : https:// doi.org/10.4000/ress.3156

Ce document a été généré automatiquement le 22 septembre 2020.

(c) Librairie Droz 


\title{
Arnault SKORNICKI et Jérôme TOURNADRE, La Nouvelle Histoire des idées politiques
}

\author{
Giovanni Carpinelli
}

\section{RÉFÉRENCE}

Arnault SKORNICKI et Jérôme TOURNADRE, 2015, La Nouvelle Histoire des idées politiques, Paris, La Découverte, 123 p.

Qu'est-ce qu'une « idée politique»? Si la question est simple, la réponse ne l'est pas. Dans l'ouvrage d'Arnault Skornicki et Jérôme Tournadre deux réponses se font face. Traditionnellement, on a été longtemps porté à croire que les «idées politiques " étaient des variations sur un petit nombre de thèmes importants et établis une fois pour toutes : l'État, la liberté, le pouvoir, et ainsi de suite. Selon une telle perspective d'ordre philosophique, le débat avait surtout pour protagonistes quelques grands auteurs classiques. Quoiqu'un peu caricaturale, cette image correspond à la démarche suivie par l'ancienne histoire des idées politiques. Depuis 1960, on assiste à un changement complet dans la manière d'aborder la discipline, telle que, près de quarante ans plus tard, une nouvelle histoire des idées politiques a pris forme qui s'est imposée à l'étranger plus encore qu'en France. C'est ce changement dont rend compte l'ouvrage.

2 Dans un premier temps, c'est surtout la méthodologie qui a été révisée. Puis l'objet même de la discipline a connu une expansion considérable. Les documents les plus divers ont fini par prendre la place des monuments - les classiques - qui dominaient la scène jusqu'alors. «Le mode d'existence des idées est pluriel » disent les auteurs dans l'introduction. Les concepts abstraits ne sont plus les seuls acteurs des idées politiques. Bien d'autres éléments sont désormais pris en considération : les croyances, les valeurs, les slogans, les représentations, les lieux communs, etc. La production des idées n'a en 
conséquence plus été perçue comme le monopole des penseurs professionnels et/ou reconnus du politique, en sorte que n'importe quel quidam pouvait tout aussi bien y jouer un rôle. D'autant que les frontières du politique sont-elles mêmes susceptibles de se déplacer et d'embrasser une grande variété d'objets sociaux: une œuvre d'art, un graffiti aussi bien qu'une discipline scientifique « véhiculent au moins implicitement une certaine vision de l'ordre social, des rapports de pouvoir, etc.» (p.4-5). Nous entrons ainsi dans un domaine beaucoup plus vaste que celui réservé à l'ancienne histoire des idées politiques. L'ouvrage entend précisément mener le lecteur à la découverte de ce monde nouveau, tout en mettant en évidence en quoi cette «nouvelle histoire des idées politiques » diffère de la conception traditionnelle de la discipline.

3 Cinq chapitres très denses font le tour des tendances les plus remarquables. La question de la méthodologie fait l'objet d'un premier chapitre. Les résultats produits par l'école de Cambridge, et notamment les travaux de Peter Laslett, John Dunn et Quentin Skinner, y font figure d'avant-garde. Parmi les promoteurs du changement, une place de premier plan revient aussi à John Greville Agard Pocock. Ce sont Pocock et Skinner qui ont montré le plus de détermination en menant une bataille sur deux fronts, contre l'idéalisme et contre le marxisme. Le maitre-mot de l'école est "contexte » : un défi $a$ priori banal (p. 9), mais qui se heurte au langage et à la rhétorique d'un milieu peu favorable à la contextualisation.

4 Le deuxième chapitre s'ouvre sur l'œuvre de Reinhart Koselleck et de ses différents collaborateurs (p. 33-41). Ici, à la différence des historiens de Cambridge, ce sont les concepts qui retiennent l'attention. Le langage et l'histoire, selon Koselleck, poursuivent une existence parallèle, ils s'éclairent mutuellement tout en restant séparés : l'histoire arrive à se passer du langage pour toute sorte de phénomènes - la naissance, l'amour, la mort, ou les maladies, la faim et la misère, etc. - et le langage à son tour ne se confond pas avec l'acte qu'il contribue à préparer, à déclencher ou à accomplir. Dans les pages suivantes, les noms de Luigi Firpo et Franco Venturi sont invoqués de manière à rendre compte de l'essor très favorable de la nouvelle histoire des idées politiques en Italie. Le sort de la France a été en revanche moins heureux, l'histoire des idées politiques ayant échoué à obtenir une reconnaissance institutionnelle et à trouver une place à part entière dans l'université et dans la recherche. L'école des Annales a joué, à cet égard, un rôle négatif en privilégiant l'histoire des mentalités. Et c'est finalement à Michel Foucault que l'histoire des idées doit son renouvellement en France. Ce dernier a voulu réaliser une généalogie du savoir: « une contre-histoire des sciences, en tant qu'elles sont imbriquées dans les systèmes de pouvoir et les luttes historiques » (p. 45). Rappelons que Foucault est avec Derrida à l'origine du tournant linguistique qui, dans le courant des années 1980, a eu une influence déterminante sur l'histoire et les sciences sociales : « comment, et à quel point, la réalité politique (l'État, les révolutions, la lutte des classes, les partis...) est-elle construite par les conventions langagières manipulées par des acteurs capables de réfléchir leur expérience et leur action?» (p. 46). En France, un tel changement est alors incarné par Jacques Guilhaumou et Pierre Rosanvallon. Le premier contribua avec d'autres, Régine Robin notamment, à introduire dans l'hexagone les travaux de l'école de Cambridge et l'histoire conceptuelle à la manière de Koselleck. Le second a construit dans les années 1970 " une œuvre abondante et polyvalente » (p. 48) qui vise à " mettre au jour les systèmes de représentation qui commandent la façon dont une société, dans sa pluralité, conduit et envisage ses actions » (p.47). En même temps, il réserve une place de choix aux idées et pense à une histoire du politique par les idées. Le tournant 
linguistique a enfin trouvé en Grande Bretagne un acteur majeur dans la figure de l'historien Gareth Stedman Jones. La conscience cesse d'être pour lui un miroir qui reflète la réalité, elle devient un facteur actif qui contribue à façonner le monde : c'est la lutte qui fait les classes plutôt que l'inverse (p. 51-52).

5 Avec les troisième et quatrième chapitres, le discours des auteurs se déroule sur un plan beaucoup plus large. Les limites temporelles reculent, on évoque la sociologie de la connaissance à partir de Karl Mannheim et l'on remonte jusqu'à Destutt de Tracy et Karl Marx pour aborder la question de l'idéologie. Les limites spatiales englobent désormais, avec l'Europe occidentale, l'Amérique du Nord. Si le centre de l'intérêt scientifique pourrait encore être désigné par le mot "contexte", il ne s'agit plus cependant du contexte langagier, intellectuel ou politique, mais du contexte « social». C'est donc une histoire sociale des idées politiques qui est envisagée par les auteurs, selon qu'il s'agisse de s'en référer aux principaux théoriciens (troisième chapitre) ou de rendre compte de l'étendue de son retentissement (quatrième chapitre).

6 Comme le montre le troisième chapitre, deux américains, le sociologue Charles Camic et le politiste Neil Gross, sont dans les années 2000 les pionniers d'une approche axée sur la dimension locale des configurations dans lesquelles se meuvent les penseurs. Un autre élément mis en valeur par les deux savants est l'identité qu'un auteur projette de lui-même (intellectual self concept). Il est montré, par exemple, à l'occasion d'un encadré, comment Gross a analysé la biographie du philosophe Richard Rorty dans un volume de près de 400 pages sur la base d'une série de traits - l'indépendance, le pluralisme, etc. qui semblent traduire la volonté de sa part d'incarner le "patriote américain de gauche» (p. 60-61). Dans un autre paragraphe, le marxisme revu et corrigé des époux Wood est à l'honneur, Ellen Meiksins et Neal Wood faisant preuve d'une finesse remarquable dans leurs études sur les différents théoriciens. L'œuvre de Pierre Bourdieu enfin est illustrée à deux reprises, respectivement dans les troisième et quatrième chapitres (p. 66-67 et p. 79-81). Foisonnant, le personnage déborde quelque peu des cases dans lesquelles on essaie de l'enfermer. Le champ intellectuel correspond chez lui au contexte proposé par l'école de Cambridge. Que son autonomie vienne à faiblir et les enjeux qui le caractérisent sont « rattrapés par la logique d'autres univers sociaux» (p.67). La sociologie des idées politiques, avec Frédérique Matonti notamment, va aussi au-delà des auteurs cardinaux pour examiner de près, à propos de la vague structuraliste notamment, le réseau varié des protagonistes mineurs, épigones, éditeurs, journalistes, soit tout un système de production. La vulgarisation se voit ici attribuer un intérêt considérable, conformément à l'enseignement de Bourdieu.

Le quatrième chapitre est centré sur l'idéologie. Après une vision rétrospective rapide, cette notion nous est présentée chez les historiens Georges Duby et Roger Chartier. L'étude des mentalités s'efface au profit d'une vision plus spécifique : tandis que Duby associe l'idéologie à l'imaginaire, Chartier essaie de remplacer l'histoire sociale de la culture par une histoire culturelle du social, telle que le domaine qui s'ouvre alors à la recherche est celui de la lutte des représentations. Bourdieu et son école sont parvenus à dépasser la référence simple à l'idéologie, en introduisant des termes qui permettaient d'explorer d'autres territoires : lieux neutres qui convergent sur des lieux communs, esprit du temps, doxa, etc. Une dernière question est enfin envisagée pour clôturer ce quatrième chapitre : comment les masses s'emparent-elles des idéologies ? Bien des transactions peuvent se produire sur le marché des biens symboliques. La 
culture des classes populaires réserve à cet égard bien des surprises et reste un domaine encore assez peu étudié.

8 Le cinquième chapitre concerne la réception éventuelle et souvent tortueuse des idées par les opérateurs sur le terrain, gouvernement, administration, personnel politique et militants. Peu de noms illustres ici et un grand éparpillement des études. Le sens des idées est soumis à des changements en fonction des contextes qui varient dans le temps et dans l'espace. La transposition donne lieu à une recréation, ainsi que le suggérait Ricoeur à propos de la traduction. Il arrive qu'une idée s'impose parce qu'elle est utile au-delà des idéologies et des intérêts en jeu. L'identification, l'invention même d'une figure nouvelle, ainsi celle du " chômeur» à la fin du XIX ${ }^{e}$ siècle, a pu permettre aux savants de se joindre aux politiques. Les sciences du gouvernement mènent à une catégorisation et à une hiérarchisation des problèmes.

Ce livre est en même temps un guide et un manifeste. En montrant ce que l'histoire des idées politiques est en train de devenir, les auteurs veulent favoriser la propagation d'un tel changement en France. Dans un pays si souvent fier de sa tradition et de son prestige intellectuel, il est frappant de constater un tel déclassement international: "plusieurs entreprises (la plupart hors de France) furent menées pour refonder le domaine et rompre avec les traditions historiographiques dominantes» (p. 3). En revanche, l'Italie s'est hissée à un rang supérieur: "terre d'élection de la pensée politique ", pays où "l'histoire de cette dernière a tôt reçu ses lettres de noblesse " (p. 41). Aussi, le but poursuivi par l'ouvrage est-il celui d'intégrer la discipline d'une façon « pleine et entière dans les sciences sociales» (p. 108). Voilà qui est dit.

D'une certaine manière, à l'attention pour la pensée en tant que telle se substitue l'attention pour le langage qui constitue et traverse la pensée. Pierre Rosanvallon, par exemple, « entend écrire une histoire de la démocratie moderne comme celle d'un sujet qui s'élabore lui-même dans l'histoire, au travers d'expérimentations, de tâtonnements et de conflits internes » (p.49). Claude Lefort, à son tour, voit dans ce régime politique l'« auto-institution du social » (ibid.). Pour Stedman-Jones, « la reconstitution du passé passe [...] par une attention soutenue aux formes rhétoriques qui contribuèrent à le produire » (p. 52). Il n'est pas étonnant que cette inflation langagière donne lieu à une dissolution de l'individu. Il est à cet égard remarquable que les encadrés explicatifs qui accompagnent les différents chapitres concernent, à l'exception de Locke et Rorty, plusieurs auteurs simultanément, renvoyés à tel ou tel de leurs ouvrages mais sans mention biographique complète. Quoiqu'une génération sépare Pocock et Skinner leurs années de naissance ne sont ainsi pas rappelées. Il en va de même pour Bourdieu et Foucault. En revanche, Meinecke, Koselleck, Croce, Firpo et Venturi ont droit à de telles précisions chronologiques. Dans un livre sur la «nouvelle» histoire des idées politiques, les trois derniers noms que nous venons de mentionner représentent, avec Gramsci, la seule production italienne bien identifiée, comme si l'apport de l'Italie à l'histoire des idées s'était arrêté là. Quelques lignes supplémentaires sur les travaux respectifs de Carlo Ginzburg, Remo Bodei ou Sergio Luzzatto n'auraient pas été malvenues. 


\section{AUTEURS}

\section{GIOVANNI CARPINELLI}

Turin, Fondazione Istituto Piemontese Antonio Gramsci 